C-A/AP/\#423

Feb. 2011

\title{
The Effects of Realistic Pancake Solenoids on Particle Transport
}

X. Gu, M. Okamura, A. Pikin, W. Fischer, Y. Luo

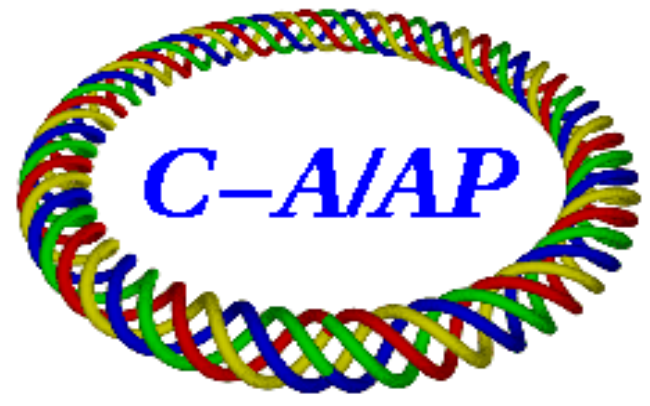

Collider-Accelerator Department

Brookhaven National Laboratory

Upton, NY 11973

Notice: This document has been authorized by employees of Brookhaven Science Associates, LLC under Contract No.

DE-AC02-98CH10886 with the U.S. Department of Energy. The United States Government retains a non-exclusive, paid-up,

irrevocable, world-wide license to publish or reproduce the published form of this document, or allow others to do so, 
 \\ The Effects of Realistic Pancake Solenoids on Particle Transport}

2 X. Gu, M. Okamura, A. Pikin, W. Fischer, Y. Luo

3 Corresponding Author: X. Gu

4 Brookhaven National Laboratory, Upton, NY 11973

\section{Abstract}

6 Solenoids are widely used to transport or focus particle beams. Usually, they are assumed as being ideal 7 solenoids with a high axial-symmetry magnetic field. Using the Vector Field Opera program, we modeled 8 asymmetrical solenoids with realistic geometry defects, caused by finite conductor and current jumpers. 9 Their multipole magnetic components were analyzed with the Fourier fit method; we present some possible optimized methods for them. We also discuss the effects of "realistic" solenoids on low energy particle transport. The finding in this paper may be applicable to some lower energy particle transport system design.

Keywords: realistic solenoids, multipole magnetic fields, particle transport

\section{Introduction}

Solenoids are used extensively for focusing and transporting the beams in modern accelerators [1-4]. Many high-energy particle detectors are equipped with superconducting (cold) solenoids [5-6], that operate at very low temperatures. Meanwhile, many normal conducting (warm) solenoids [7-10] work at room temperature.

To evaluate the effects of a solenoid on the beam's parameters, we must calculate the magnetic field [11] or transfer matrix [12-14]. Many researchers discussed ways of assessing magnetic fields, wherein the fields are calculated on-axis [15] or off-axis [16], with an infinite solenoid [17-19], semi-infinite one [20], or a finite one [21-22]. Some papers and textbooks concentrate on calculating the transfer matrix [12-14]. When evaluating either of them, static magnetic fields produced by cold or warm solenoids usually are assumed to be axially symmetric.

Nevertheless, many applications [23-24] require at the least an estimate of the solenoid's multipole magnetic fields with asymmetry, caused by the realistic solenoid structure. However, very few papers cover this situation.

Moreover, although some effort [23-25] was devoted to ascertaining the magnetic fields of a solenoid's multipole components, it is important thoroughly to study the origins of these components, and to devise methods of reducing them using the structure of the coil winding.

The pancake-type solenoid is a frequent choice for applications requiring inexpensive high-power density. Their popularity mainly rests on the ability to connect its electricity in series and the water flow in parallel. 
is reduced by 180 degrees by rotating the interval pancakes in this solenoid. This finding may be applicable to transporting several species of lower energy, such as electrons [26-28], protons [29] and ions [30-34], or for emittance compensation [23-24, 35] in photocathode electron guns.

We begin with a brief overview of warm solenoid structures, and then analyze the solenoid magnetic fields with different structures. After that, we discuss the effects of one and two "realistic" solenoids on the propagation of particle beams. Finally, some conclusions and recommendations for further study are presented.

\section{4}

\section{Structure of the Pancake Solenoid}

The multipole magnetic field components of realistic solenoids are caused by their asymmetrical construction, which includes cross over angles, transition angles, pancake polarity, leads, and pancake rotation.

Pancake is the basic element of this kind of solenoid. One warm solenoid is constructed by assembling several pancakes in different combinations. Fig. 1 shows the geometric structure of one pancake.
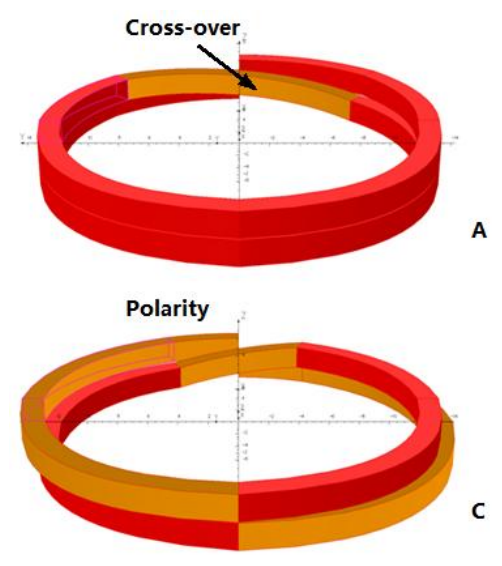

C

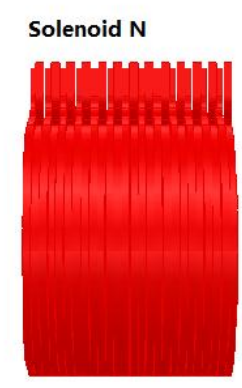

E
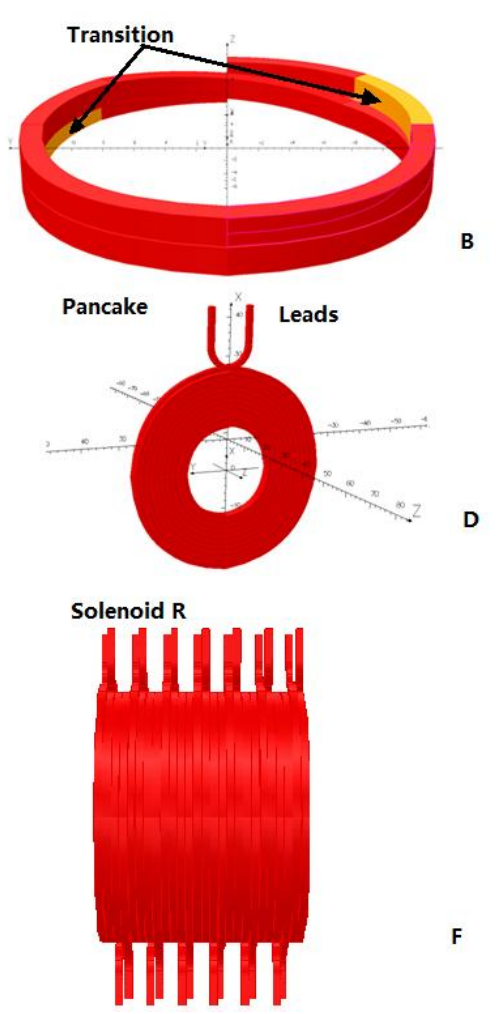

Fig. 1 Structure of a Pancake and Solenoid

One pancake consists of two spirals joined on their inner radius by a cross over (Fig. 1 A). Here, we define the cross over angle as the half angle of the cross over conductor. Fig. $1 \mathrm{~A}$ and Fig. $1 \mathrm{C}$ have, respectively, a $45^{\circ}$ and a $22.5^{\circ}$ cross over angle.

Each layer of the pancake's spiral is almost a circle. A transition conductor connects one layer to another one (Fig.1 B). The first inner layer must transit to the second layer before reaching the cross over 
conductor. The transition is defined from the transition angle to the cross over angle. The transitions in Fig. $1 \mathrm{~B}$ and Fig. $1 \mathrm{C}$, respectively, start from $90^{\circ}$ to $45^{\circ}$, and $180^{\circ}$ to $22.5^{\circ}$.

Each pancake can have its own polarity. Compared with Fig.1 B, Fig.1 C not only has a different cross over and transition angle, but also has an opposite polarity. After winding several layers, the resulting pancake with leads is illustrated in Fig.1 D.

One solenoid can have many pancakes that may have different polarities or different rotations. The solenoid named Solenoid $\mathrm{N}$ has same pancake polarity and rotation direction (Fig. $1 \mathrm{E}$ ); the solenoid called Solenoid $\mathrm{R}$, has same pancake polarity but alternate pancake rotation (Fig. $1 \mathrm{~F}$ ). In this paper, six of 13 pancakes are rotated by $180^{\circ}$ alternately in Solenoid $\mathrm{R}$. Solenoids that have same pancake rotation but alternate pancake polarity are named Solenoid $\mathrm{P}$.

Accordingly, the solenoid with different cross over, transition angle, pancake polarity and pancake rotation can have different multipole magnetic field distributions. Furthermore, the leads of individual pancakes may affect these distributions.

\section{Analysis Method}

Using the different geometric parameters discussed in Section 2, we constructed several realistic solenoids modeling them via Vector Field Opera program. All have thirteen pancakes, each pancake with 10 layers. Their inner- and outer- diameters are $234 \mathrm{~mm}$ and $526 \mathrm{~mm}$, respectively. Their length is $379.6 \mathrm{~mm}$. Fig. 2 illustrates the distribution of the longitudinal magnetic field strength of one of them.

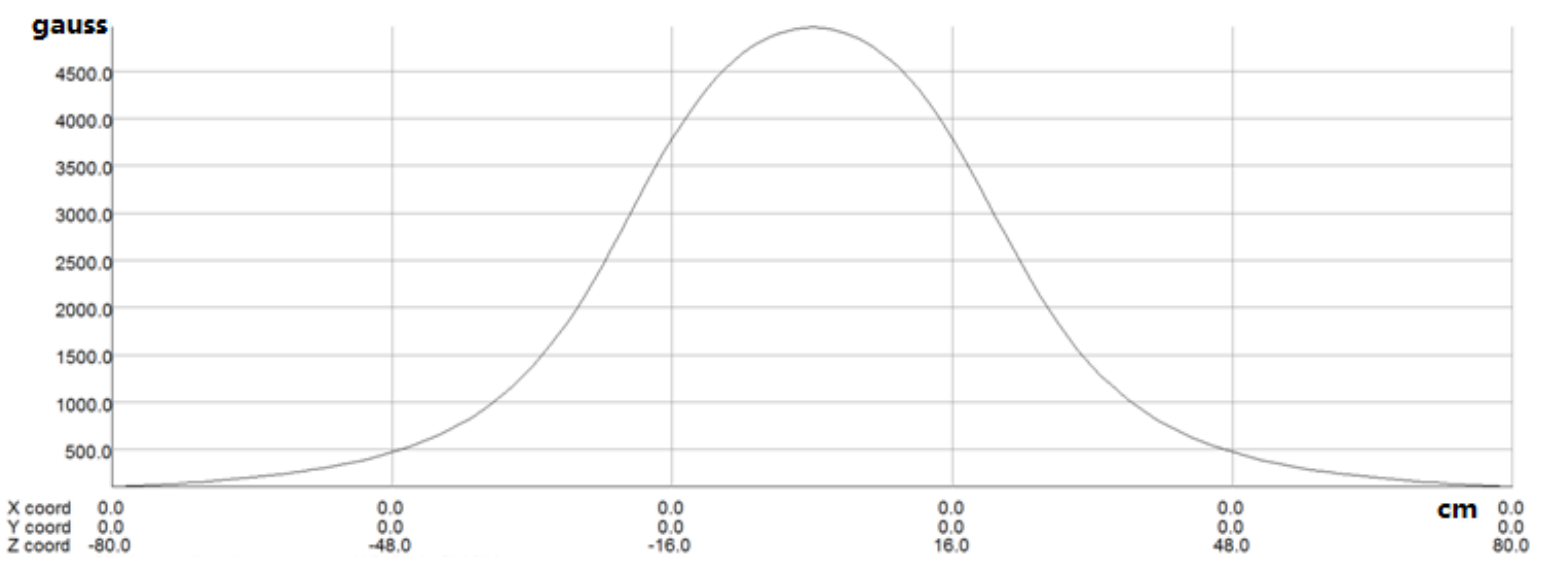

Fig. 2 Solenoid and Its Longitudinal Field Bz

The multipole magnetic field components generated by such "realistic" solenoids are analyzed and compared by Fourier fit method.

In cylindrical coordinates, we can express the radial and azimuthal components of magnetic field B in the form [36-37]:

$$
\begin{aligned}
& \mathrm{B}_{\mathrm{r}}(\mathrm{r}, \theta)=\sum_{n=1}^{\infty}\left(b_{n} \sin (\mathrm{n} \theta)+a_{n} \cos (\mathrm{n} \theta)\right) \\
& \mathrm{B}_{\theta}(\mathrm{r}, \theta)=\sum_{n=1}^{\infty}\left(b_{n} \sin (\mathrm{n} \theta)-a_{n} \cos (\mathrm{n} \theta)\right)
\end{aligned}
$$


Where $b_{n}$ is the amplitudes of the $2 n$ pole normal term and $a_{n}$ is the amplitudes of $2 n$ pole skew term in the "European Convention".

85 The multipole magnetic field, $B_{\theta}$ can be computed on a reference radius $R_{r e f}$ at different longitudinal positions and fitted as Fourier series. Then, according to formula (2), the coefficients of this Fourier series are the multipole magnetic field components. The reference radius $R_{r e f}=75 \mathrm{~mm}$, and the longitudinal position from $-80 \mathrm{~cm}$ to $+80 \mathrm{~cm}$ are used in this paper. The original point of cylindrical coordinate was set at the center of the solenoid's geometry.

\section{Multipole Components for Different Solenoid Structures}

In this section, we calculate the normalized multipole magnetic field components for different solenoid structures. They are the solenoids with different leads, transition angles, cross over angles, pancake rotations and pancake polarities.

Figure 3 shows the multipole components for solenoids with and without leads.

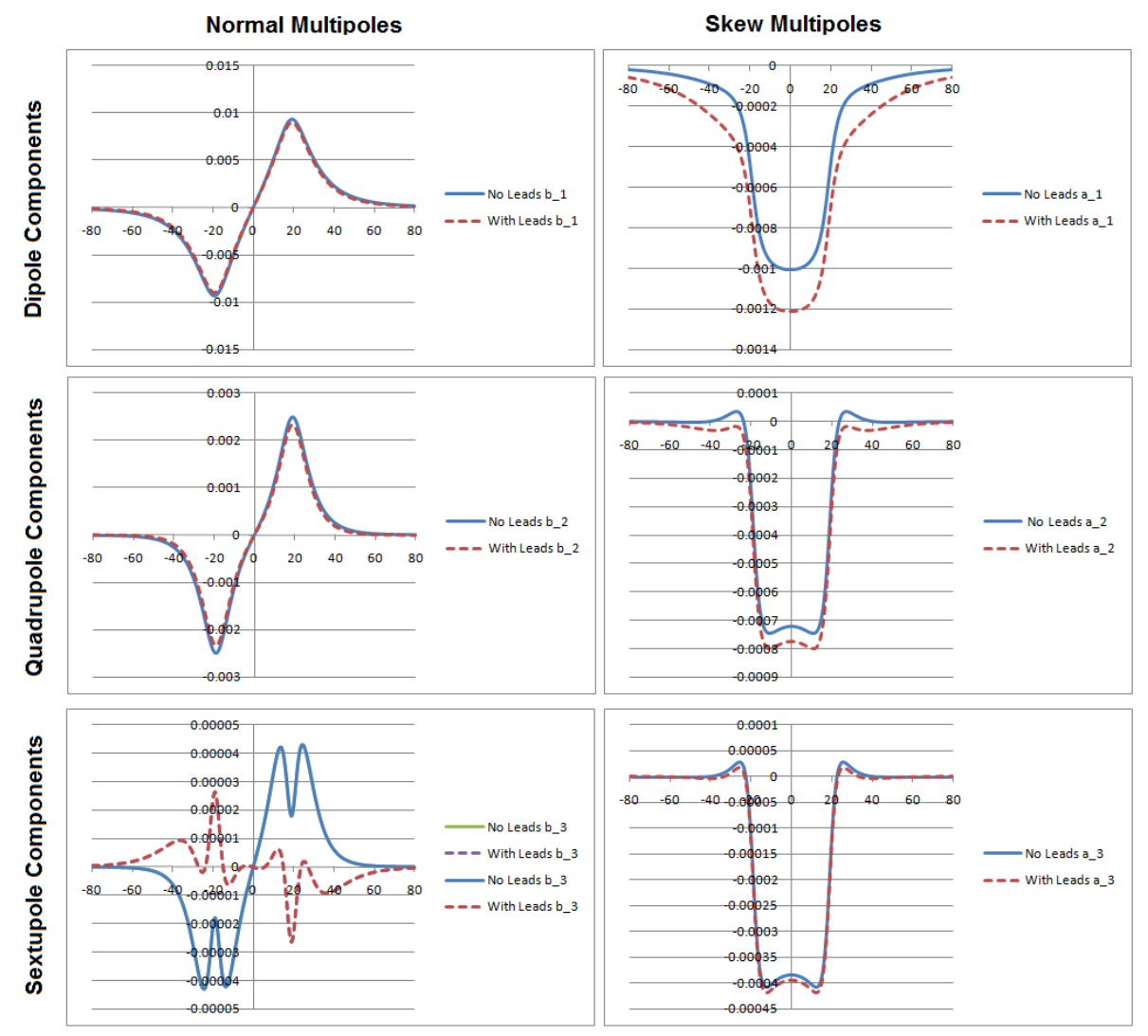

Fig. 3 Solenoids Multipole Components with and without Leads

In Fig. 3, the three rows respectively represent the dipole, qaudrupole, and sextupole components; the two columns represent the normal (left) and skew (right) multipole components. The horizontal axis is the longitudinal position in units of centimeters and the vertical axis is the normalized amplitudes of the multipole component. Because the multipole components with $n>3$ have lower magnetic field strength, only those with $n \leq 3$ are shown. 
As evident from Fig. 3, the distribution of multipole component $(n \leq 3)$ with and without leads have only a slight difference except for the normal sextupole magnetic field. For this reason, in further analyses we removed the leads from the models.

Figure 4 plots the calculated solenoid multipole components with different transition angles.

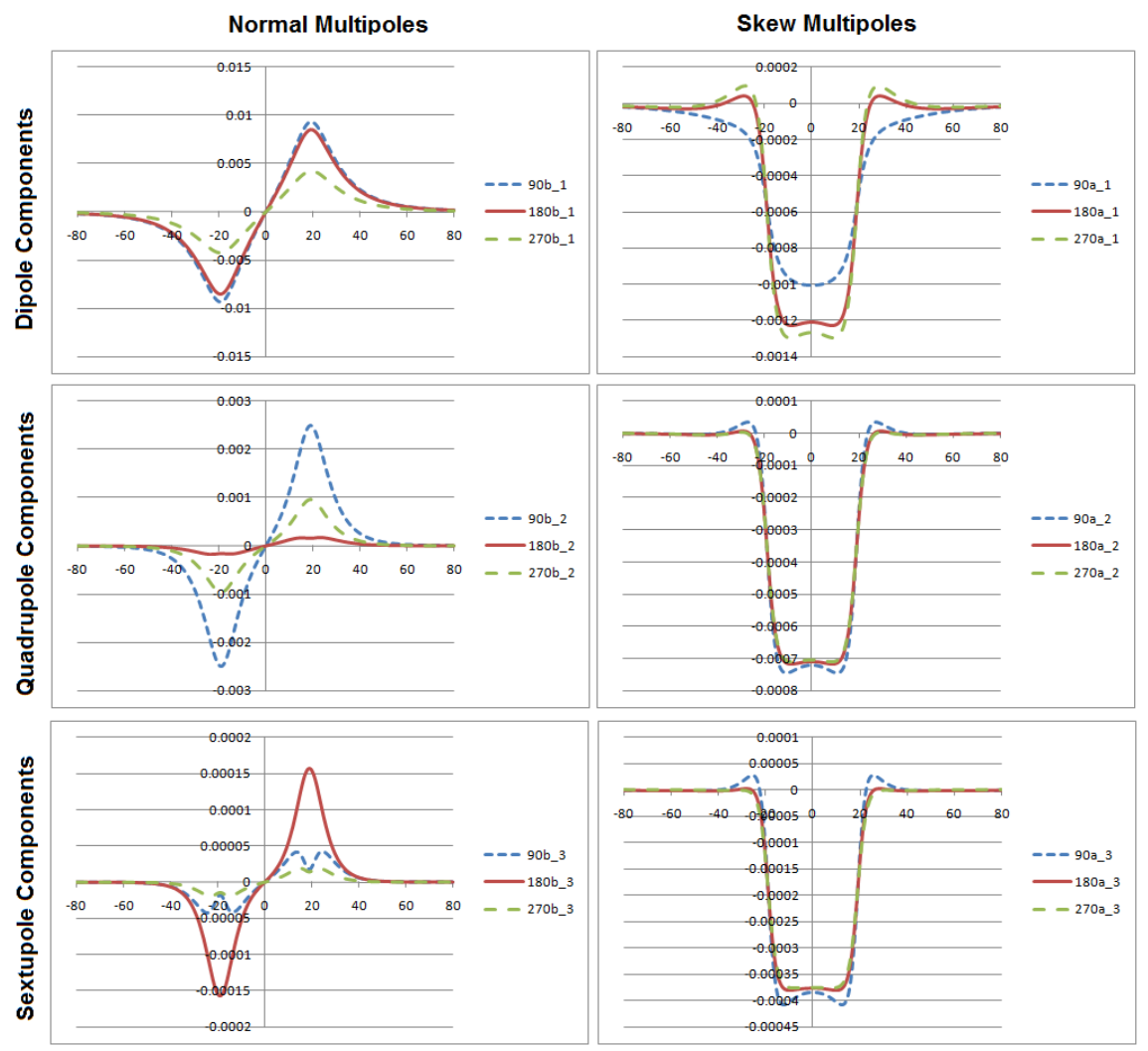

Fig. 4 Solenoids Multipole Components versus Transition Angles

With the same cross over angle $\left(22.5^{\circ}\right)$, we studied three solenoid structures with $90^{\circ}, 180^{\circ}$ and $270^{\circ}$ transition angles. Fig. 4 reveals that different transition angles induce different distributions of high order component. Seemingly, the solenoid with the $270^{\circ}$ angle has the minimum normal dipole component, while the solenoid with the $180^{\circ}$ angle has the minimum normal quadrupole component.

The multipole components of solenoids with different cross over angles were computed and are plotted in Fig. 5. 


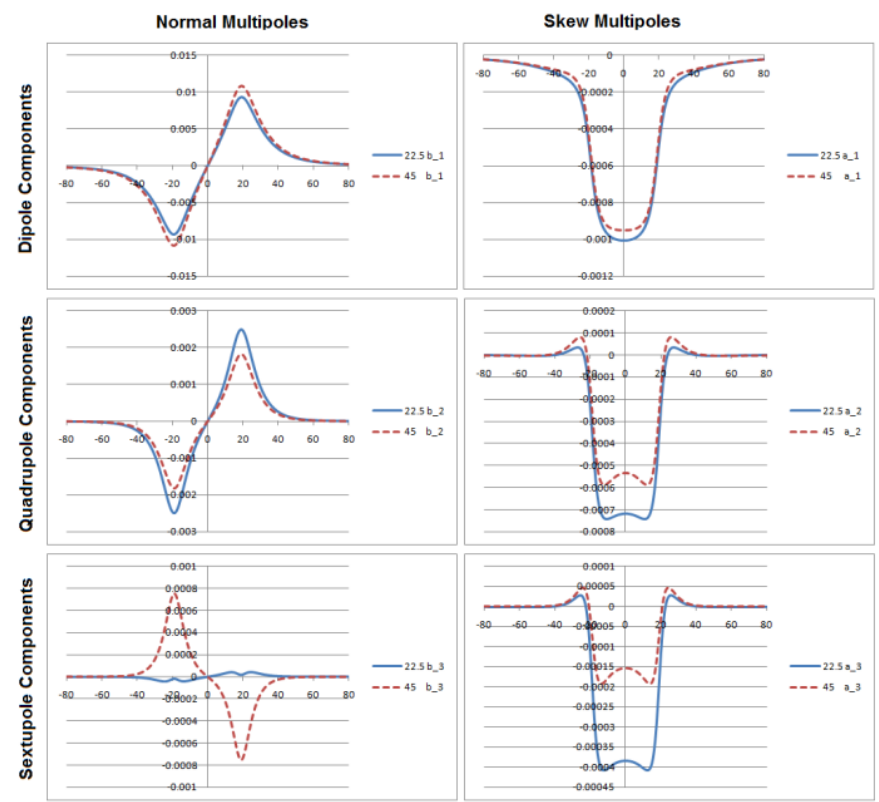

Fig. 5 Solenoids Multipole Components versus Cross over Angle

116 In this instance, we changed the cross over angle from $22.5^{\circ}$ to $45^{\circ}$ and transition angle is $90^{\circ}$. Because 117 conductor transits from the transition angle to the cross over angle, the length of transition conductor 118 also changes. The effects of the cross over angle on multipole components are unclear.

119 Figure 6 plots the calculated multipole components of solenoids with (Solenoid R) and without (Solenoid $120 \mathrm{~N})$ pancake rotation. The transition is set to $270^{\circ}$ and the cross over angle is $22.5^{\circ}$.

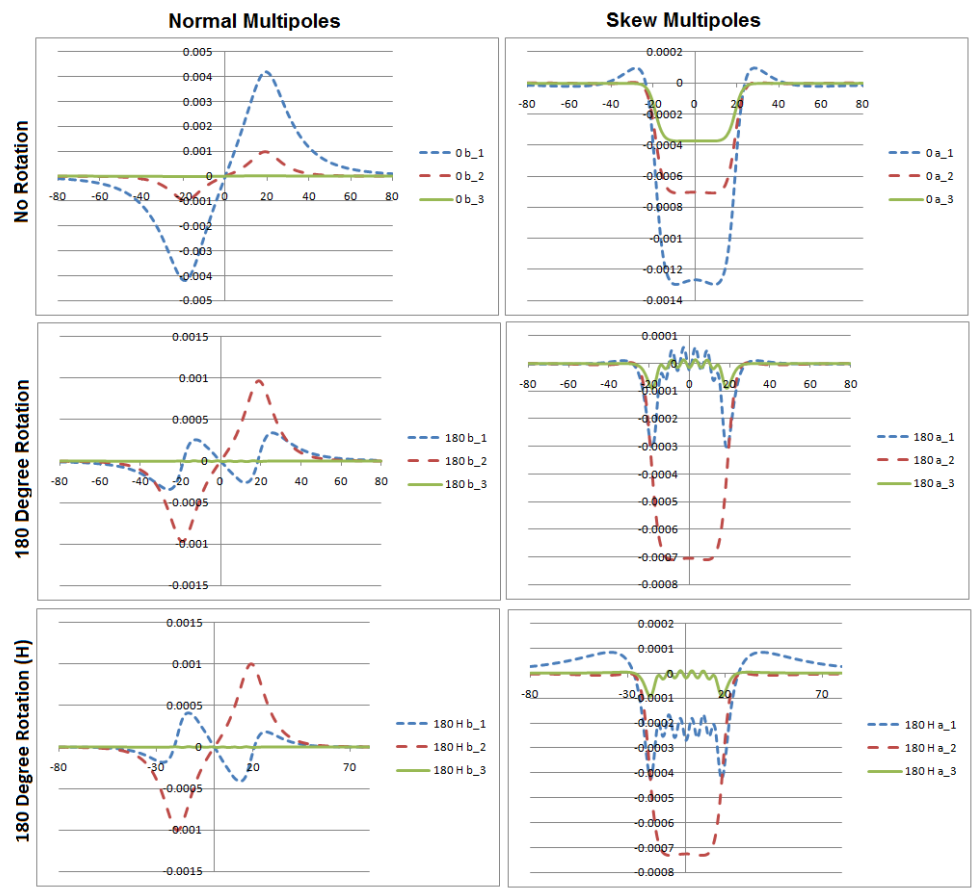

Fig. $6180^{\circ}$ Rotation versus no Rotation 
124 In Fig. 6, b (a)_1, b (a) _2 and b (a) _3 correspond, respectively, to normal (skew) dipole, quadrupole and sextupole components. The first row is calculated with Solenoid $\mathrm{N}$ and the second with Solenoid R.

126

127

128

129

130

From second row in Fig. 6 , we conclude that the $180^{\circ}$ alternate rotation of pancakes in a normal solenoid reduces the normal dipole component dramatically. This result is confirmed by the solenoid with the $180^{\circ}$ transition angle and $22.5^{\circ}$ cross over angle. However, apparently it does not change the normal qaudrupole and sextupole components.

Nevertheless, for Solenoid R, it is difficult to assemble a coil with output wires pointing in opposite directions. Besides, there should be some jumpers connecting these coils in series, and they also will introduce some multipole components.

To resolve this problem, we changed the layers of seven non-rotated pancakes in Solenoid $\mathrm{R}$ from 10 to 9.5 , so that all pancakes have the same azimuthal position. The simulation for this configuration is shown as the third row in Fig. 6.

The normal dipole component can be reduced by using Solenoid $\mathrm{R}$ with a $180^{\circ}$ rotation. The quadrupole component can be optimized by $90^{\circ}$ pancake rotation or $270^{\circ}$ rotation; in this instance, the transition angle is set to $90^{\circ}$ and the cross over angle is $22.5^{\circ}$.

From Fig. 7, we note a reduction in both the normal quadrupole component and normal dipole component. The skew quadrupole component also decreases, but the skew dipole component increases.

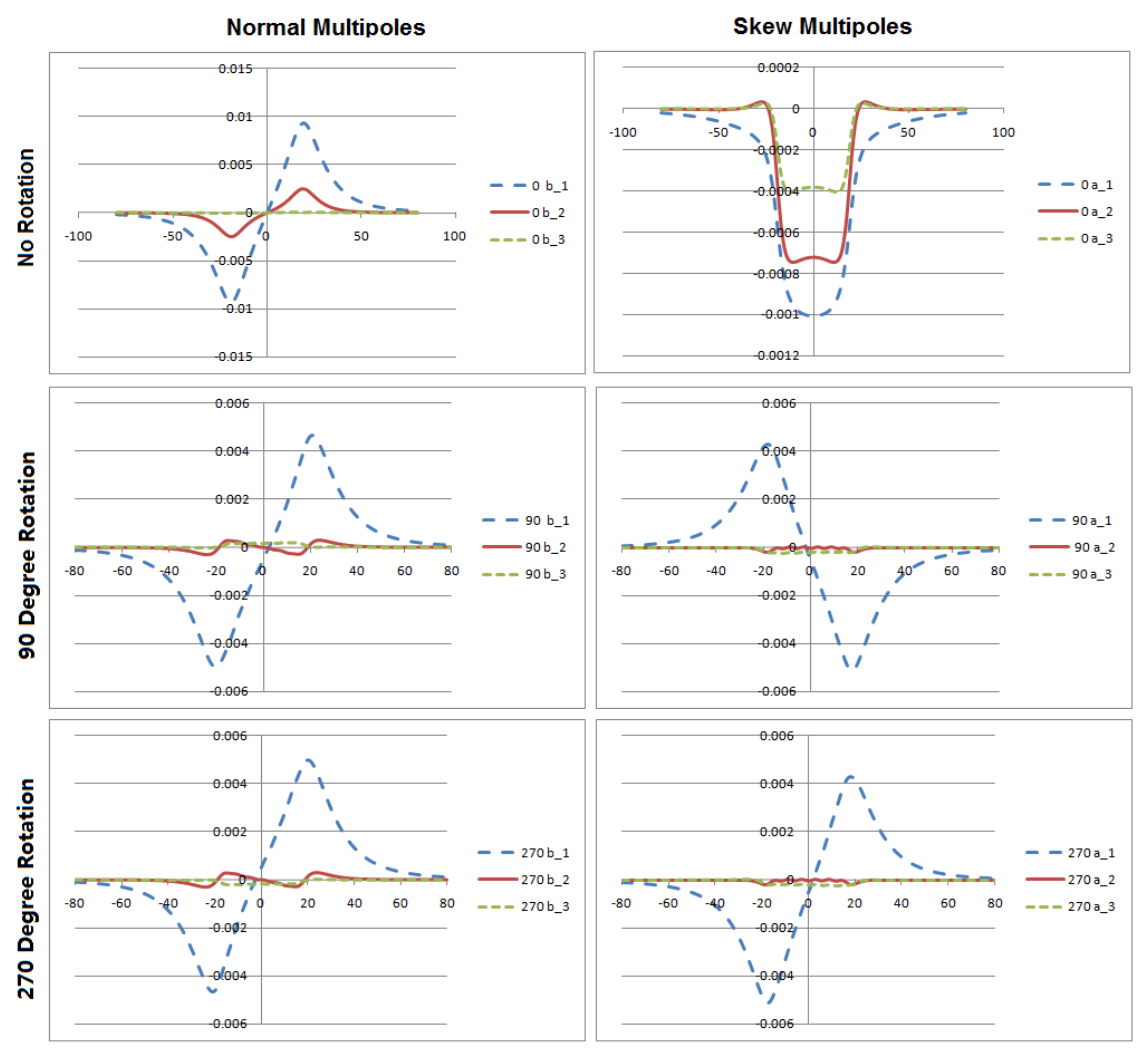


Finally, the components of the solenoid multipole magnetic field with (Solenoid P) and without (Solenoid $\mathrm{N})$ polarity pancakes, were assessed and are presented in Fig. 8.
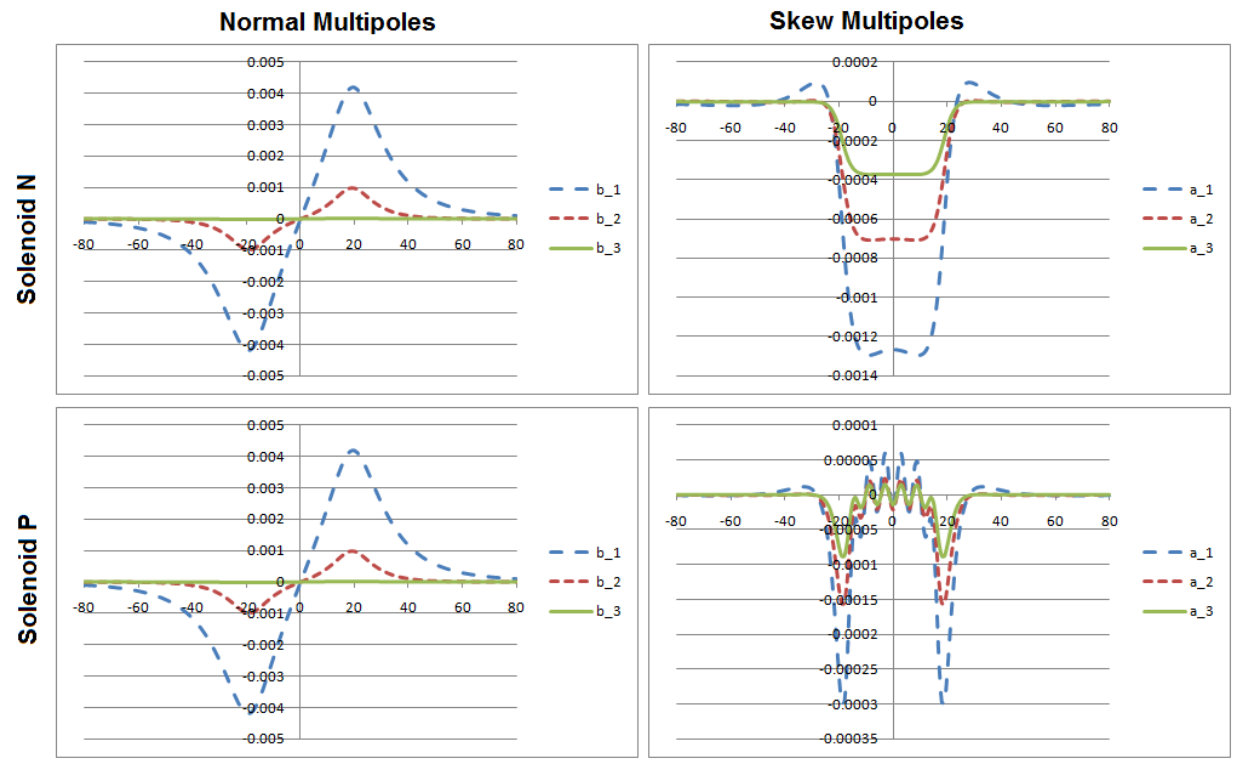

Fig. 8 No Polarity versus with Polarity

This figure shows that in Solenoid $\mathrm{P}$ only the skew multipole components change; the normal multipole components do not.

According to different design requirements, we can optimize the normal dipole, quadrupole, and sextupole components and skew the dipole components by using different transition angles; thereafter further optimization is achieved different pancake rotation.

\section{Beam Transport with a Realistic Solenoid}

In this section, firstly, we discuss the detrimental effects of a single realistic solenoid on particle transport. Then we analyze the effects of two realistic solenoids on propagation of a particle beam.

The dipole component's field of a single realistic solenoid can deflect some kinds of particle trajectories at low energy. To verify this effect and find methods to improve it, we simulated the passage of some particles through a single Solenoid $\mathrm{N}$ and Solenoid $\mathrm{R}$. These solenoids have the same dimensions, with a $180^{\circ}$ transition angle and a $22.5^{\circ}$ cross over angle.

Table 1 Position Change for Different Particles

\begin{tabular}{llll}
\hline Particle & Atomic Number & Charge & Energy (kV) \\
\hline Proton & - & +1 & 80 \\
$\mathrm{H}$ & 1 & -1 & 50 \\
$\mathrm{He}$ & 2 & +1 & 13 \\
$\mathrm{C}$ & 6 & +5 & 102 \\
$\mathrm{Ne}$ & 10 & +2 & 22 \\
$\mathrm{Si}$ & 14 & +13 & 238 \\
$\mathrm{Fe}$ & 26 & +20 & 442 \\
\hline \hline
\end{tabular}




\begin{tabular}{llll}
\hline $\mathrm{Au}$ & 79 & +32 & 493 \\
\hline
\end{tabular}

160 The center of solenoid is set at $Z=0$, and their axis is oriented along the Z-axis. All particles start from $Z=-$ $16125 \mathrm{~cm}$ on the solenoid axis. Table 1 lists the particle's parameters; their trajectories are illustrated in Fig. 1629.
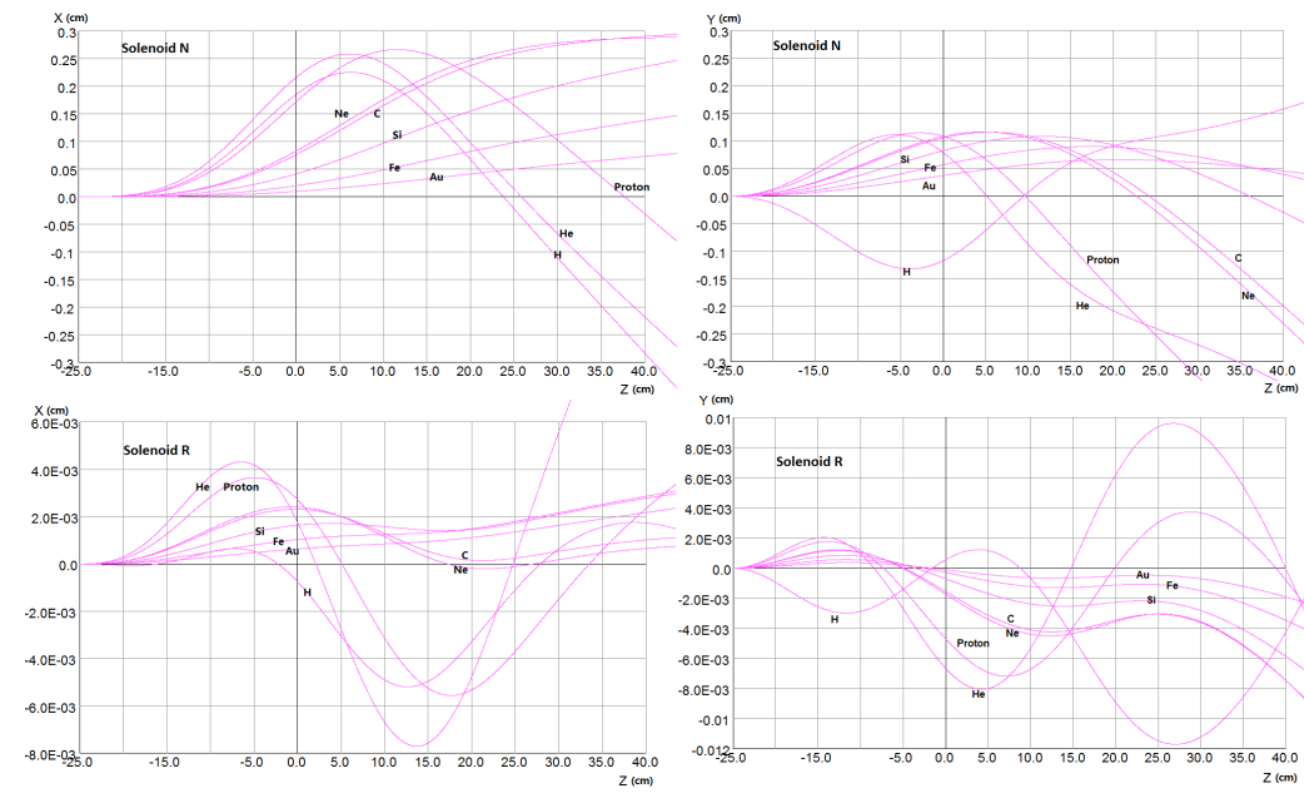

Fig. 9 Particle Trajectories with Normal and Rotated Solenoid

As Fig. 9 reveals, unlike the ideal solenoid, the particle's trajectories do not follow along the center axis when they pass through these realistic solenoids. From $-25 \mathrm{~cm}$ to $40 \mathrm{~cm}$, the particle trajectories of Solenoid R have at least 10 times smaller deviations in transverse amplitude than do those of Solenoid $\mathrm{N}$.

Sometimes, the beam transport system has several contiguous solenoids. We discuss the effects of relative solenoid orientation on beam propagation for Cases A and Case B in Fig. 10 that have only two 170 adjacent realistic solenoids.

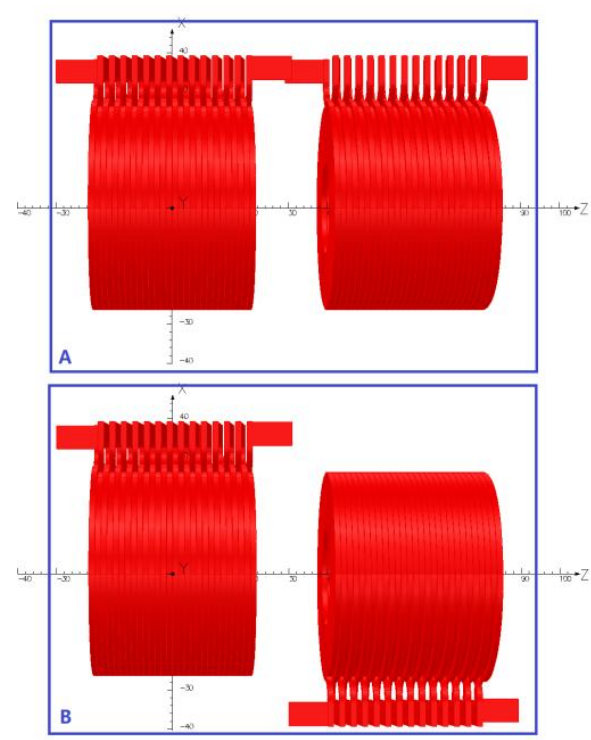


173

174

175

176

177

For Cases $A$ and $B$, we use two kinds of solenoids, Solenoid $N$ and Solenoid $R$; their configurations are termed Case A-N, Case A-R, Case B-N, and Case B-R, respectively. The center of left solenoid is set to $Z=0$, another solenoid's center is set to $Z=60 \mathrm{~cm}$. In the simulations, a single electron starts from $Z=0$, and its velocity is parallel to the solenoid's axis. The simulated trajectories for these four cases are shown in Fig. 11.

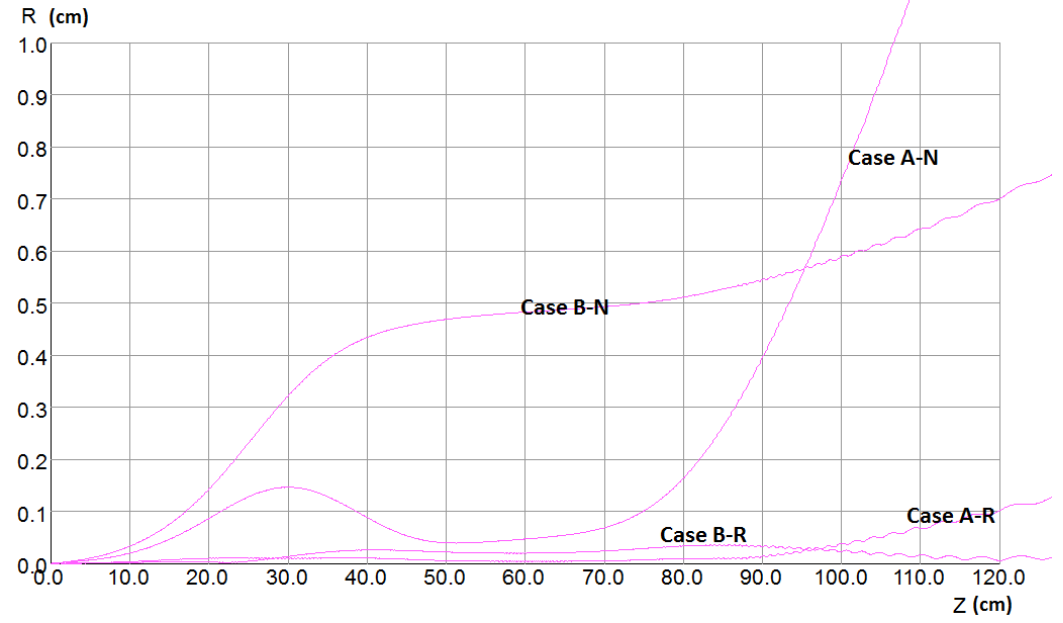

Fig. 11 Trajectories for Different Solenoid Arrangements

As depicted, Case B undergoes less angle change after passing through two solenoids than Case A; while for both cases with Solenoid $\mathrm{R}$, there is less change in angle and position than Solenoid $\mathrm{N}$. Thus, when designing a low energy beam transport system with solenoids, different beam requirements maybe necessitate different solenoid configurations.

\section{Discussion}

In this paper, we presented some simulations with realistic solenoids and their affects on the transport of low energy particle beam. Unlike the ideal solenoids, the realistic solenoids have high order components that can deflect particle's trajectories. With the $180^{\circ}$ alternate rotation of pancakes in the normal solenoids, the normal dipole component can be reduced dramatically. Using these solenoids (Solenoid R), we can design a low energy particle's transport system with less angle and position deviation. Combined with the transfer maps [39], which include high order multipole field, these simulations also can help us in understanding the particle's trajectories when they pass the realistic solenoids.

However, there are more researches are needed on this topic. Firstly, the effects of cross over angles are not very clear. Secondly, because there is a force placed on wire when winding the pancakes, the final realistic transition angle and cross over angle may differ from their design value. This force may distort them, and introduce more complex conductor geometry in pancakes. More research also is needed on quadrupole and sextupole effects. 
199

200

201

202

203

204

205

206

207

208

209

210

211

212

213

214

215

216

217

218

219

220

221

222

223

224

225

226

\section{Acknowledgment}

The authors acknowledge helpful discussions with Walter Shaffer and Jonathan Hock.

\section{References}

[1] A. Yamamoto, Y. Makida, R. Ruber, et al., Nucl. Instr. and Meth. A 584 (2008) $53-74$.

[2] S.H. Aronson, J. Bowers, J. Chiba, et al., Nucl. Instr. and Meth. A 499 (2003) $480-488$.

[3] S. Seletskiy, in: Proceedings of PAC07, Albuquerque, New Mexico, 2007, pp: $3109-3111$.

[4] A.A. Efremov, E.K. Koshurnikov, Y.Y. Lobanov, et al., Nucl. Instr. and Meth. A 585 (2008) $182-200$.

[5] L. Mirabito, Nucl. Instr. and Meth. A (2010), doi:10.1016/j.nima.2010.02.243

[6] D. Toprek, Y. Nosochkov, Nucl. Instr. and Meth. A 612 (2010) $260-273$.

[7] S.J. Russell, Z.-F. Wang, W.B. Haynes, Phys. Rev. ST Accel. Beams. 8 (2005) 080401.

[8] R.J . Hayden, M.J . Jakobson, Nucl. Instr. and Meth. A 278 (1989) 394 - 396.

[9] S. M'Garrech, Nucl. Instr. and Meth. A 550 (2005) 535 - 542.

[10] E. Beebe, J. Alessi, S. Bellavia, et al., Rev. Sci. Instrum. 71 (2000) $893-895$.

[11] D. B. Montgomery, Solenoid Magnet Design, John Wiley \& Sons, Inc. New York, 1969.

[12] G. Xu, Phys. Rev. ST Accel. Beams. 7 (2004) 044001.

[13] D. Rubin, in: Alexander Wu Chao and Maury Tigner (Eds.), Handbook of Accelerator Physics and Engineering, 2nd ed., World Scientific Publishing Co. Pte. Ltd. Singapore, 2002, pp.272 - 277.

[14] G. Franchetti, Phys. Rev. ST Accel. Beams. 4 (2001) 074001.

[15] C. Chia and Y. Wang, Phys. Teach. 40 (2002) $288-289$.

[16] R. H. Jackson, IEEE Trans. Electron Devices 46 (1999) 1050 - 1062.

[27] V. Labinac, N. Erceg, D. Kotnik-Karuza, Am. J. Phys. 74 (2006) 621-627.

[18] O. Espinosa and V. Slusarenko, Am. J. Phys. 71 (2003) 953 - 954.

[19] K. Fillmore, Am. J. Phys. 53 (1985) 782-783.

[20] G. V. Brown and L. Flax, J. Appl. Phys. 35 (1964) 1764-1767.

[21] J. Farley and R.H. Price, Am. J. Phys. 69(2001) 751 - 754. 
[22] V. I. Danilov, M. Ianovic, Nucl. Instr. and Meth. 94 (I971) $541-550$.

228 [23] M. Ferrario, M. Migliorati, P. Musumeci, et al., in: Proceedings of EPAC 2006, Edinburgh, Scotland, 2292006, pp. $169-171$.

230 [24] J. Schmerge, LCLS Gun Solenoid Design Considerations (LCLS-TN-05-14), LCLS project, SLAC, 2005.

231 [25] A. Wolski, M. Venturini, in: Proceedings of EPAC2004, Lucerne, Switzerland, 2004, pp: 2185 - 2187.

232 [26] V. Shiltsev, K. Bishofberger, V. Kamerdzhiev, et al., Phys. Rev. ST Accel. Beams. 11 (2008) 103501.

233 [27] D. Xiang, Y.C. Du, L.X. Yan, et al., Phys. Rev. ST Accel. Beams. 12 (2009) 022801.

234 [28] W. Fischer, Y. Luo, A. Pikin, etc, in: Proceedings of IPAC10, Kyoto, Japan, pp: $513-515$.

235

[29] G. Ciavola, L. Celona, S. Gammino, et al., in: Proceedings of LINAC2002, Gyeongju, Korea, 2002, pp: $674-676$.

237 [30] E. Lee, Nucl. Instr. and Meth. A 544 (2005) 187 - 193.

238 [31] R. Hollinger, P. Spädtke, Rev. Sci. Instrum. 79 (2008) $02 B 704$.

239 [32] J. Alessi, D. Barton, E. Beebe, et al., in: Proceedings of LINAC2006, Knoxville, Tennessee, USA, 2006, 240 pp: $385-387$.

241 [33] M. Eshraqi, G. Franchetti, A.M. Lombardi, et al., Phys. Rev. ST Accel. Beams. 12 (2009) 024201.

242 [34] J.H. Li , J.Y. Tang, Nucl. Instr. and Meth. A 574 (2007) $221-225$.

243 [35] J.B Rosenzweig, A.M Cook, M.P. Dunning, et al., in: Proceedings of 2005 Particle Accelerator 244 Conference, Knoxville, Tennessee, 2005, pp. 2624-2626.

245 [36] A. K. Jain, in: Turner, S [ed], CAS - CERN Accelerator School : Measurement and Alignment of 246 Accelerator and Detector Magnets, Geneva, CERN, 1998, pp. 1- 26.

247 [37] A. K Jain, P. Wanderer, in: Alexander Wu Chao and Maury Tigner (Eds.), Handbook of Accelerator 248 Physics and Engineering, 2nd ed., World Scientific Publishing Co. Pte. Ltd. Singapore, 2002, pp.409 - 414.

249 [38] J. Back, J.Pozimski,P. Savage, et al, in: Proceedings of IPAC10, Kyoto, Japan, pp: 648 - 650.

250 [39] C. E. Mitchell and A. J. Dragt, Phys. Rev. ST Accel. Beams. 13 (2010) 064001. 